\title{
Un nuovo tipo di magnetometro per le misure magnetiche sulle rocce
}

\author{
R. Cialdea
}

Ricevuto il 6 Luglio 1966

\begin{abstract}
Riassunto. - JiA. deserive un movo tipo di magnetometro, realizzato nell'Istituto Nazionale di Geofisica, particolarmente indicato per il rilevamento della direzione dell'intensitit di magnetizzazione, eventualnente presente nelle rocce ignee. Niella nota viene slaborata la teoria dell'apparato e ne vengono deseritte le caratteristirlif tecnicbe o la sensibilita; si suggerisce infine la teenica da seguire per il rilevamento della direzione dell'in. tensità di magnetizzazione delle rocee.
\end{abstract}

Summir. .. 'Tle A. describes a new magnetometer realized in the "Istituto Nazionale di Geofision", particularly appointed to determine the direction of the maguetizat iom intenity, bvantually presert in the igneous rocks. In the presint paper the A. wabolates the theory of this apparatus and describes its techmial rharacteristics and its sensibility: at last the A. proposes the tereluical metlod in order 10 motermine the direction of the magnetization intensity.

Le misure sul matgnetismo delle rocet presentano delle notevoli difficoltà dato il valore genteralmente picteolo della loro milgnetizzaziont. I maguetometri rostruiti a tutt'ograt $\left(^{1}\right)$ risultano particolamemle delicati e solo in aleuni mes ("on particolari areorgimenti essi possono essepe usati in mo nomaly laboratorio di ula grande città Per questa ragione ho eerato di realizzari un ntovo tipo di magnetometro che mi permettesse di ragrimigere sensibilità e precisione elevate, pur essendo dotato di notevoli anchlteristiche di semplicità " di robustezza: esso è particolarmente indieato per il rilevamento della direzione dell'intensiti di magnetizzazione.

Il magnetometro da me realizzato consistr in una base di legno vincolata al oscillare in un determinato piano verticale per mezzo di due sospensioni bifiluri. Un telaio in bachelite si trova al disotto della 
base ed in esso il campione di roccia in ssame puo essere blocerto in una determinata posizione prefissata. Il telijo con il campion può essere ruotato intomo ad un asse verticale (ncllat posizione di riposo del pendolo), mentre un'alidada indica la rotazione esegutat.

Nella sua oscillazione il campione passa sopra a due bobine $B_{1}$ e $B_{2}$ in modo ohe il piano di oscillazione $\pi$ contenga il punto di mezzo $M$ della congiungente i centri $C_{1}$ e $C_{2}$ delle duo bobine e sia ad essa normale (v. Fig. 1). Le diw bobine, opportunamente dimensionate, sono poste in

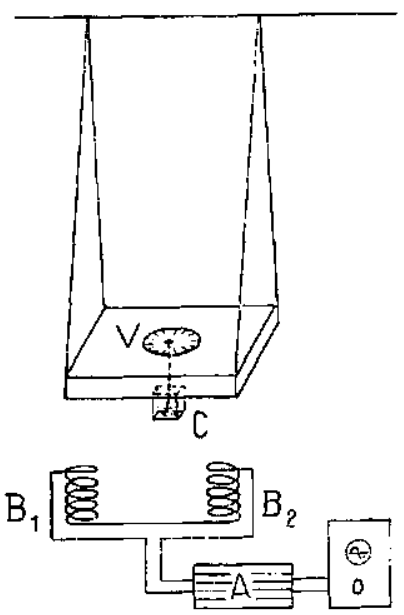

[ig. ]

opposizione in modo che sia nulla ogni f.e.m. generata da eventuali variazioni di campo magnetico purehò esse possano essere considerate uguali in $C_{1}$ e in $C_{2}$. Il che es corlaneme verificato per le variazioni del c.m.t. e quasi sempre jere lo valuzioni dol t.m. dovnte a correnti vario nel Jaboratorio, a meno che nonl si ponga lapparaio molto vicino a davi percorsi da correnti. Se i due campi (in $C_{1} e^{2}$ in $C_{2}$ ) sono variabili nel tempo e diversi, all'uscita delle due bobine si aveì un segnale, che, passando abtraverso l'mulifinctore $A$, viene inviato ad un oscillografo. Ad ogni oscillazione il cimpione $C$, se ì manetizzato, genera um campo magnetico, che assume in ganert valori diversi in $C_{1}$ e in $\mathrm{C}_{2}$. Con una opportuna rotazione del campione $O$ si può fare in modo che i due campi in $\mathrm{C}_{1}$ e in $\mathrm{C}_{2}$ siano praticannente nguali istante per istante: in questo caso il segnale in uscita dalle due bobine sarà ovviamente nullo.

Dopo questa rapida descrizione del magnetometro passerò a studiare le sue caratteristiche principali: nel paragrafo a) tratterò della 
forma e delle dimensioni delle due bobine, con le loro caratteristiohe elettriche e magretiche; nel parasrafo b) calcoleró il ampo $B$ nei due centri delle duo bobine; net parayrafo e) studiero la forma del segnale; nel paragrafo a) parteró della teeniea segnita per il rilevamento della direzione di matgnetizzazione delle roces.

a) Le BoBrie

Le bobine devono concatenare al massimo il flusso del vettore $B$, ma nello stesso tempo devono ovviamente lasciale libero dello spazio per permettere il passagrgio dal campione. La prima*idea è stata quella di costruire delic bobine' sferiche, che grodono della proprietà che il flusso del vettore $B$ ad esse collcatenato ì proporzionale al valore che la componente del vettore $B$ Iungo l'asse della bobina assume al centro della bobina stessa. Si è preferito invece costruire delle bobine eilindriche di opportune dimensioni, che gorlono di analoga proprietà. Si è trovato infatti $\left(^{2}\right)$ che per una bobina dindriog delle seguenti dimensioni ( $\mathrm{v}$. Fig. 2)

$$
L=0,72 D \quad \text { e } \quad d \leqslant 0,33 D
$$

il flusso concatenato del vettore $B$ è proporzionale al valore che assume al centro della bobina la componente del vettore $B$ lungo l'asse della bubina stesse.

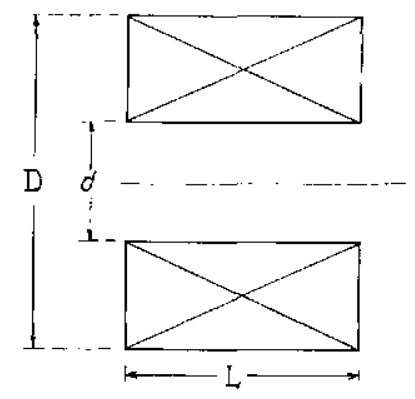

Fig. 2

Si trovano così le seguenti caratteristiche per una tale bobina: numero totale delle spire

$$
N=0,24 \frac{D^{2}}{\delta^{2}}
$$


resistenza elettrica

$$
R=0,640 \frac{D^{3}}{\delta^{4}}
$$

coeff. di autoinduzione

$$
L=0,211 \frac{D^{5}}{\delta^{4}} \quad \text { nel sistcma assoluto e.m. }
$$

flusso concatenato

$$
\phi(B) \div 9,1 \cdot 10^{-2} \frac{D^{4}}{\delta^{2}} \cdot B_{l}
$$

dove si è indicato ton $\delta$ e con o rispettivarnente il diametro del filo e la resistività olettrica del matepiale costituente il filo stesso.

Le bobine da me realizzate hanno i seguenti valori

$$
L=56,8 \mathrm{~mm} \quad D=79 \mathrm{~mm} \quad a=26,5 \mathrm{~mm} \quad \delta=0,115 \mathrm{~mm}
$$

e le sue caratteristiche teriehe sono

$$
\begin{aligned}
N & =113.280 \text { spire } \\
R & =33.900 \Omega \\
L & =3,71 \cdot 10^{11} \text { u.d.m. : : } 371 H \\
\phi(B) & =2,68.10^{-6} B_{l} \text { u.e.un. } \\
\frac{\phi(B)}{B} & =2,68 \cdot 10^{-2} \text { weber/ganss } .
\end{aligned}
$$

In realtà le bobine da me costruite presentano le seguenti caratteristiche leggermente diverse da quelle teoriche:

$$
\begin{aligned}
N & =91.300 \text { spire } \\
R & =31,5 K \Omega \\
L & =300 \mathrm{H} \\
\frac{\phi(B)}{B} & =2,17 \cdot 10^{-\approx} \text { weber/gauss } .
\end{aligned}
$$

Ai capi delle due bobine si ha quirdi una f.e.m. data da

$$
e=-\frac{d}{d t}\left[\phi\left(B_{1}\right)-\phi\left(B_{2}\right)\right]
$$


e sostituendovi i valori del magnetometro

$$
e=2,17 \cdot 10^{-2} \cdot \frac{d}{d t}\left(B_{l_{1}}-B_{l_{2}}\right)
$$

dove $e$ è espresso in volt, $B$ in ganss ad il tempo $t$ in secondi.

b) CALCOI,O DI $\left(B_{l_{1}}-B_{l_{2}}\right)$

Si stabilisca anzitutto una terma di assi di riferimento $O, x, y, z$, sinistrorsa con l'origine nel centro geometrico del campione di roccia (supposto abbastanza piccolo ed uniformemente inagnetizzato), quando si trova nella posizione di equilibrio, con l'asse $z$ lungo la verticale verso il basso, con l'asse $x$ parallelo alla congiungente i centri $C_{1}$ e $C_{2}$ delle due bobine e con l'asse $y$ ad essi normale; in questo modo il piano di oscillazione coinciderà con il piano $y z$.

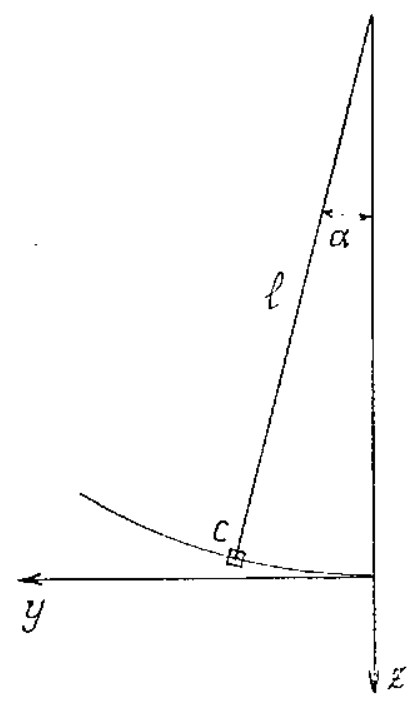

Fig. 3

In un generico istante il campione ha le coordinate $0, Y, Z$, e se si introduce l'angolo $\alpha$ che l'asse del pendolo passante per il campione forma con la verticale, si ha anche (Fig. 3)

$$
Y=l \operatorname{sen} \alpha \quad Z=l(\cos \alpha-1)
$$


dove $l$ è la Junghezzal del pendolo, rioè lal distanza del cumpione dal centro di sospensione. I "«ntri delle clue bobine hammo invece le coordinate

$$
C_{1} \equiv\left\{\begin{array}{l}
x=a \\
y=0 \\
z-h
\end{array} \quad C_{2}-\left\{\begin{array}{l}
x:=-a \\
y-0 \\
z=h
\end{array}\right.\right.
$$

Si consideri ora una nova terma di assi cartesiani $\Omega, \xi, \eta, \zeta$ solidale alla base oscillinte e coincideute con la terna $0, x, y$, z nella posizione di riposo del pesudolo. Sial $\vec{m}$ il momento magnetico del campione

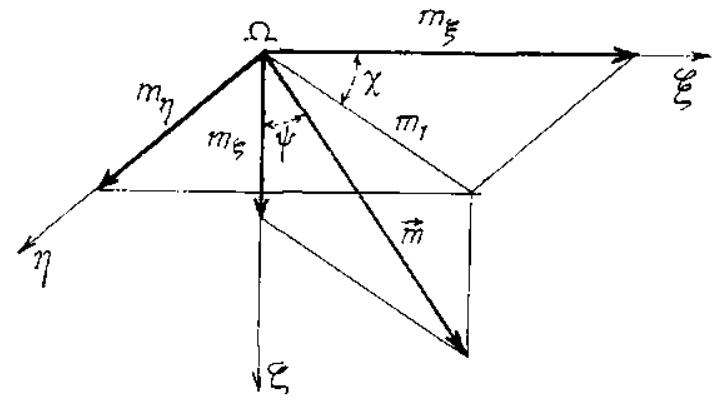

Hig. 4

in esame orientato come in Figr. 4: le (omponenti di questo momento rispetto agli assi $\xi, \eta, \zeta$ somo

$$
\left\{\begin{array}{l}
m_{\xi}=m \operatorname{sen} \psi \cos \psi \\
m_{\eta}=m \operatorname{sen} \psi \operatorname{sen} \psi \\
m_{\zeta}=m \cos \psi
\end{array}\right.
$$

In una generiea posiziote del pendolo vandlerizzata da un generico valore $\alpha$ dell'angolo formato dal perdolo con la verticale (v. Fig. 3) le componenti del momento magnetiroo $m$ risjecto algli assi $x, y, z$ sono

$$
\left\{\begin{array}{l}
m_{x}=m_{\xi}-m \operatorname{sen} \psi \cos \chi \\
m_{y}=m \cos \psi \operatorname{sen} \alpha+m \operatorname{sen} \psi \operatorname{sen} \chi \cos \alpha \\
m_{z}-m \cos \psi \cos \alpha-m \operatorname{sen} \psi \operatorname{sen} \chi \operatorname{sen} a .
\end{array}\right.
$$

Pertanto il campo milguetico genesto dal campione in un punto generioo di coordinate $r, y, z$ è dato dalle sentlenti espressiomi, dove 
$0, Y, Z$, sono le coordinate del centro del campione, cioè le coordinate dell'origine $\Omega$ della terna solidale con la base

$$
\begin{aligned}
& H_{x}=\frac{m_{x}}{R^{3}}-3 x-x m_{x}+\left(Y-\frac{y}{)} m_{y}+(Z-z) m_{z}\right. \\
& R^{5}
\end{aligned}
$$

dove si è indicato con $R$ la distanza di $\Omega$ dal punto considerato, cioè

$$
R=\left[x^{2}-(Y-y)^{2}+(Z-z)^{2}\right]^{1 / 2}
$$

Delle tre componenti di $H$ interessa solo la componente parallela all'asse delle bobine, cioè la $H_{2}$. Nei due punti $C_{1}$ e $C_{2}$ la $H_{2}$ assume i seguenti valori:

$$
\begin{aligned}
& H_{1, z}=-\frac{m_{z}}{R^{3}}-3 h m_{x} a+Y m_{y}+(Z-h) m_{z} \\
& R^{5} \\
& H_{2, z}=\frac{m_{z}}{R^{3}}-3 h-m_{x} a+Y \frac{m_{y}}{R^{5}}+(Z-h) m_{z}
\end{aligned}
$$

Ma per calcolare la f.e.m. si dovià fare la differenza tra questi due valori:

$$
H_{1, z}-H_{2, z}=-\frac{6 h a \cdot m_{x}}{R^{5}}
$$

questa espressione diventia, introducendovi lo [2]

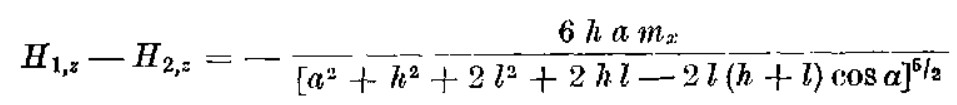

che può essere messa sotto la forma

$$
H_{1, z}-H_{2, z}=-\frac{6 h a m_{2}}{[A-G \cos \alpha]^{5 / 2}}
$$

dove

$$
A=a^{2}+h^{2}+2 l^{2}+2 h l \quad \text { e } \quad G=2 l(h+l)
$$

sono delle aratteristiche geomelpiche dell'apparato. 


\section{c) LA FORMA DEL SEGNALE}

La f.e.m., considerando delle piccole oscillazioni, per le quali si può porre

$$
a=\alpha_{o} \operatorname{sen} \omega t
$$

diventa, esprimendo $B$ nel sistema assoluto $0 . m$.

$$
e=3,2 \cdot 10^{-1} \cdot \ldots+\frac{h a \alpha_{o} G \operatorname{sen} \alpha}{(A-G \cos \alpha)^{2 / 2}} \cdot \frac{a^{2-}}{a_{o}^{2}}-m_{x}
$$

Si può constitare quindi che il segnale è proporzionale alla componente del momento magnetico normale al piano di oscillazione.

Dalla [5] si vede inoltre che la $e$ è funzione del tempo attraverso I'angolo $\alpha$; tale f.e.m. dovrà ovviamernte presentare almeno un massimo che potrà essere calcolato aunulando la derivata della funzione

$$
f(\alpha)=-\frac{\operatorname{sen} \alpha}{[A-G \cos \alpha]^{7 / 2}}
$$

Si può ord osservare ehe l'angrolo $a_{\mathrm{n}}$ ger il quale si dovrà averte il valore massimo della $e$ dovrà essere molto piecolo: infatti tale massimo dovrà aversi presumibilmente quando il campione si troverà ad unk distauza dalla posizione di riposo direa uguals al ruggrio della bobina. Nel magnetometro da me realizzato si ha

$$
a_{\mathrm{M}} \simeq \frac{r}{l}=\frac{4}{400} \simeq 10^{-\mathrm{n}}
$$

Pertanto sarà possibile sviluppare in serie le funzioni trigonometriche che appaiono nella [6], triscurando le potenze superiori ad $\alpha^{2}$; si ottiene così:

$$
a_{M}^{2} \simeq \frac{a^{2}+h^{2}}{6} \frac{h^{2}}{l^{2}}
$$

Per questo valore la $f(\alpha)$ diventa

$$
f\left(\alpha_{M}\right)=-\frac{0,238}{l\left(a^{2}+h^{2}\right)^{3}}
$$


Il valore massimo della f.e.m. divernta quindi

$$
e_{M}=0,15\left[\begin{array}{c}
h a \\
\left(a^{2} \div \overline{\left.h^{2}\right)^{3}}\right.
\end{array}\right] \sqrt{g l \cdot \alpha_{0} \cdot m_{x}}
$$

dove lio sostituito il valore della os del pendolo.

La grandezza tra parentesi dipentle dalla posizione delle bobine rispetto al campione e, datio la simmetria dolla funzione, tale grandezza assumerà il valor massimo per $a=h$; in questo caso si ha

$$
e_{M}=1,92 \cdot 10^{-2} \sqrt{g l} \cdot \alpha_{0} \cdot \frac{m_{x}}{a^{4}}
$$

Pes il magnetometro da me realizzito si hal, assendo $l=1 \cdot 10^{2} \mathrm{~cm}$, $a=8 \mathrm{~cm}, \alpha_{o}=0,7$,

$$
l_{M}=2,1 m_{x}=2,1 * H_{x} \quad(m V)
$$

dove $v$ ed $M_{x}$ sono rispetivamente il rolune e la componente doll'intensità di masnetizzazione dol campiune in esane nella direzione dell'asse $x$; poichè si possono adoperale campioni aventi un volume di circa $30 \mathrm{~cm}^{3}$, si pluo avere, se $H$ lat lia dirajone deil'asse $x$

$$
e_{M}=70, H \quad(m \mathrm{~V})
$$

La f.e.m. assumerà quindi il valore massimo $e_{\mathrm{M}}$ dato dalla [7], ma è opportumo vedere la sua variazione nel tempo; a questo seopo seurà sufficiente fare il rapporto tra la f.e.m. per m generico valore di $\alpha$ e la $e_{M}$. Si ottiene cosi

$$
\frac{e}{e_{M}}=\frac{f(\alpha)}{f\left(a_{M}\right)}=\frac{a}{a_{M}} \cdot\left(\frac{a_{o}^{2}-\alpha^{2}}{a_{o}^{2}-a_{M}^{2}}\right)^{1 / 2}\left[\begin{array}{l}
A-G\left(1-\frac{a_{M}^{2}}{2}\right) \\
A-G\left(1-\frac{\alpha^{2}}{2}\right)
\end{array}\right]^{1 / 2}
$$

con la solita approssimazione; ma, essendo inoltre $\alpha_{0} \gg a_{M}$ si puó porre anche

$$
\frac{e}{e_{M}}=\frac{a}{a_{M}}\left[\frac{A-G\left(1-\frac{\alpha_{M}^{2}}{2}\right)}{A-G\left(1-\frac{\alpha^{2}}{2}\right)}\right]^{7 / 2}
$$


Il generico valore di $\alpha$ si può ora esprimere in unità $a_{M}$, cioè si può porre $\alpha=k \alpha_{\mathrm{K}}$, da cui

$$
\begin{aligned}
& A-G+G \frac{a_{M}^{2}}{2}-\left(a^{2}+h^{2}\right) \frac{7}{6} \\
& A-G+G{ }_{2}^{\alpha^{2}}=\left(a^{2}+h^{2}\right) \frac{6+k^{2}}{6}
\end{aligned}
$$

il rapporto e/e risulta così molto semplificuto

$$
e_{u}^{e}=k\left|\frac{7}{6+k k^{2}}\right|^{p / 2}
$$

Nella Fig. 5 ho riportato la toma del segrale che si ottiene; in ascisse è riportato il tempo in mità $\tau$, equivalente al tempo necessario al pendolo per pereoruere l'angolo $a_{\mathrm{M}}$, riè

$$
\tau=\frac{a_{\underline{M}}}{a_{0} \omega}
$$

ed in ordinate $\dot{e}$ riprostato il rapporto $e / e_{\mathrm{Mr}}$.

Il segnale consiste quindi in una serie di impulsi della forma indicatid nella Fig. o, intervallati di un tempo $\Delta t$ dato da $\pi \sqrt{l / g}$; nel mio magnetometro si ha $\Delta t \simeq 2^{\prime \prime}$.

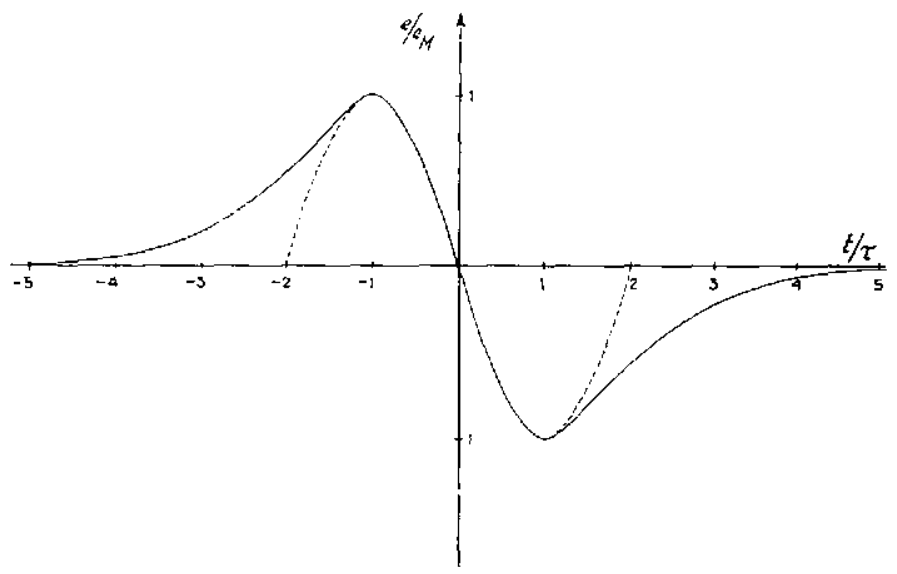

Frig. 5

Ma ciascun impulso può essere ritenuto in una prima approssimazione come una sinussoide di periodo uguale al doppio di $\tau$, cioè al doppio della distanza tra il massimo positivo e quello negativo: nella Fig. 5 ho riportato a puntimi la simussoide cosi calcolatia. 
La frequenza di un tale segnale, supposto intlefinito, è allora

$$
v=-\frac{1}{2 \tau}=-\left.\frac{a_{0}}{2 a_{M}}\right|^{\prime} \frac{\bar{g}}{l}=\frac{\sqrt{3} \alpha_{0}}{2 a} \sqrt{g l}
$$

Nel nostro caso si lua

$$
v=72 H_{z}
$$

\section{a) RIIEYAMENTO JELLA DIREZIONE DI MAGNETIZZAZTONE}

Si supponera ethe il aumpiome sia magnetizato unifolmemente di modo che il momento mitmetico sara rapperesentato da un vettore passante per il centro geometrico del rampione. In tal caso si traced sul ampione una tersta di assi $\varrho$, $5 . \eta$. :omunqua collegata con l'orientamento geografico rlclla localitì dalla quale è stato prelorato. Si ponga ora il campione nel telaio del mannetometro in modo che wli assi $\xi, \eta, \zeta$ siano coincidenti rispettivannente con lasse $x, y=z$ (v. Fig. 1). Nel masnetometro allobia si arrà un saprale proporzionale a

$$
m=-m_{5}=m \operatorname{sen} \psi \cos \%
$$

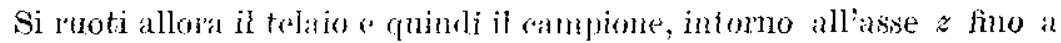

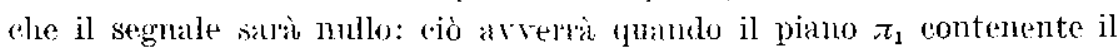

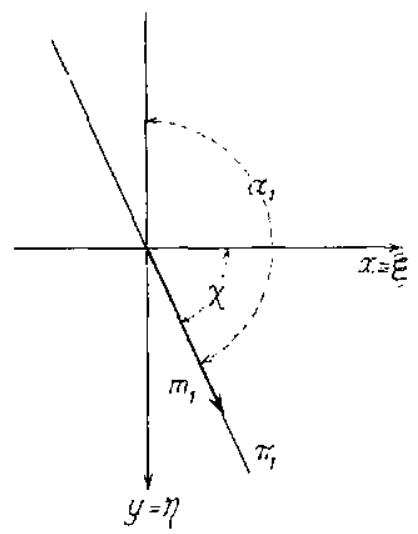

Fig. 6

vettole $m$ (Fig. 6), che nella posizione iniziale formava un angolo $\chi$ con i'ssse $x$, sarà ruotato di un angolo $\alpha_{1}$ in verso antiorario, dato da $a_{1}==90^{\circ}+\chi$.

Si ponga ora il canpione nel telaio, in modo che gli assi $\xi, \eta$ e $\zeta$ coincidano rispettivamente con gli assi $x, z e-y$. Nel magnetometro si avrà aneora un segnalo properzionale a $m_{x}=m_{\xi}=m \operatorname{sen} \psi \cos \%$. 
Si ruoti ora il telaio e quindi il campione intorno all'asse $z$, cioè intomo all'asse $\eta$ fino a rendere nulo il sechnale; ciò av verrà quando il piano $\pi_{2}$ contenente il vettore $m$ (Fig. 7 ) sarà di nutovo normale all'sse $x$; cioè si dovià avere

$$
\operatorname{ton} \beta_{1}=\frac{m_{\xi}}{m_{5}}=\operatorname{tg} \psi \cos \chi
$$

dovè $\beta_{1}$ è l'angolo di cul si è dovuto ruotal' il telitiu.

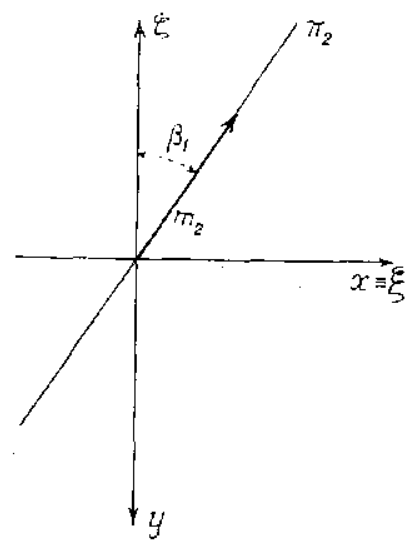

lig. 7

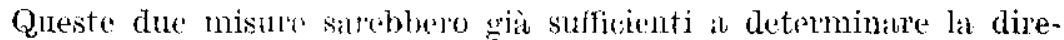
zione di magnetizzazisue del amplone relativinente alle coordinate geografiche, poichè con uso si sullo misumati grli angoli $\psi$ e $\chi$. Ma è ovvio we il cumpione potrebbe oriantarsi nel talaio in m modo qualunque; nolla tabella ho liportato i 24 modi principali di orientaunento del campione, indicando le direzimi en i versi ahe possono avere le tre com-

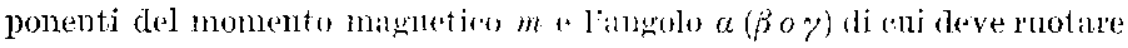

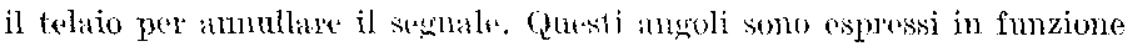

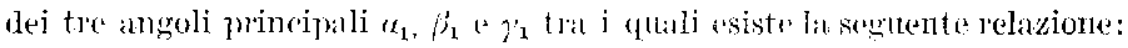

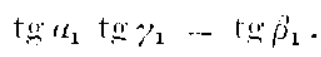

Inoltre essi sono collegali alle cautteristiche natgretiche del eampione secondo le sementi aspuessioni

$$
\begin{aligned}
& \operatorname{tg} \alpha_{1}=-\frac{m_{\xi}}{m_{\eta}}=\operatorname{ttg} \% ; \\
& \operatorname{tg} \beta_{1}=l_{m_{5}}^{m_{5}}=\cos \psi \operatorname{tg} \psi \quad ; \quad \operatorname{tg} \gamma_{1}=\frac{m_{\eta}}{m_{\zeta}}-\operatorname{sen} \psi \operatorname{tg} \psi
\end{aligned}
$$


Tiellultima colonnat ho riportato il valore della componente del momento nuignetico mel piiuno orizontale (nella posizione di riposo del pendolo); dai sto valore dipende la semsibilitì dell'apparato. Infatti siat $m_{i}$ il valore di tale romponente: il segnale avid il valore massimo

$$
e_{M}=1 m_{i} \operatorname{sen} i
$$

dore $A$ è ma costante dell'apparato (v. la [i]) a l'indice $i$ individua uno dei tre gruppi di orientamento possibili del ampione. L'angolo $\lambda$ è quello formato dalla componrnte $m_{i}$ ron il piano di oscillazione $\pi$. Sia ora $\sigma$ la sensibiliat dell'apmaratu, cioè il valor ninimo apprezabbile dellat f.e.m. e, si ha

$$
\sigma=A m_{i} \hat{m}_{\mathrm{min}}
$$

dove $\lambda_{\mathrm{m} i n}$ è il minimo ansulo di rotazione del talaio, della posizione di zero, che può essete percepito dall'apparito.

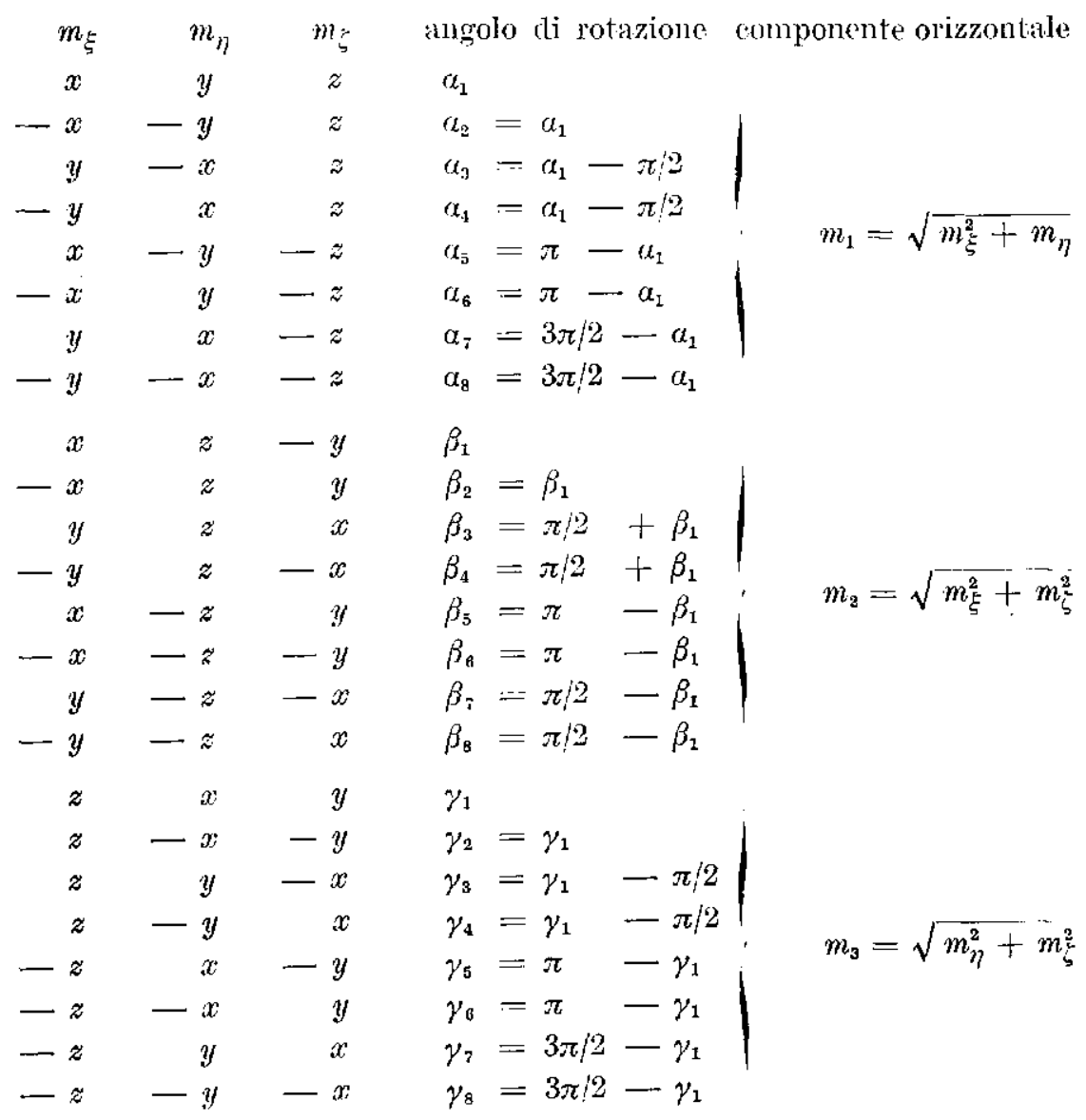


Cosi la massima semsibilitì por langolo $\hat{\lambda} \dot{e}$

$$
\lambda_{\text {min }}=-\frac{\sigma}{d} m_{i}
$$

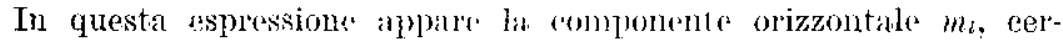
tamente inferiore al momento manntico $m$ del ampione; ma si vede subito ehe, essendo suffirenti solo due orientazioni possibili del campione, si potranno scegliere quelle orientazioni che rendono le componenti orizzontali di $m$ pì̀ grandi possibile. Il caso peggiore si avrà quando $m$ è orientato in modo da rendere uguali le tre componenti $m_{\xi}, m_{\eta}$ e $m_{\zeta}$ di $m$. In questo raso si ha $m_{\xi}=m_{y}=m_{\zeta} \simeq 0.8 m$, da cui

$$
\lambda_{m}=0, \frac{\sigma}{A m}
$$

dove $A m$ è il valore massimo del seglatle, quando $m$ è nomnale a $\pi$ : esso è dato dalla [8] e quindi, nel nost jo caso

$$
\lambda_{m}=\frac{\sigma}{60 \mathrm{H}}
$$

Con ma intensità di magnetizzizione di $10^{-3}$ ed una sensibilità di $\check{\text { s }} \mu \mathrm{V}$ si ha

$$
\lambda_{m}=4^{\circ} 40^{\prime}
$$

mentre con una intensità ai nadrnetazzazione di $10^{-2}$ la sensibilità può magriungere i $28^{\prime}$ circa.

Queste sunsibilità possono essere notevolnudute migliorate se invece di cercare la posizione di segnali nullo, si determina il valore massimo della $e$ per vari angoli $\lambda$, in modo da rilevare la courva $e_{M}=e_{M}(\lambda)$, che risulta essere la sinussoide [11], o, contando gli angolj della posizione iniziale, rioè ponendo $\lambda=\varphi-\mu$

$$
e_{M}=A m_{i} \sin (\varphi-\mu)
$$

dove $\lambda$ è l'angolo che $m$ forma eon il piano di oscillazione $\pi, \varphi$ è l'angolo di cui bisogna ruotare il campione per annullare il segnale (cioé

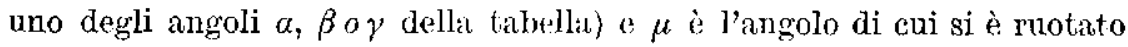


il campione della posizione iniziale (v. Fig. 8). Si può così applicare il metodo dei minimi quadrati e si trova, determinando per es. Ia $e_{M}$, corrispondente a diversi valori di $\mu$ intervallati di $10^{\circ}$,

$$
\operatorname{tg} \varphi=\frac{1,26 \frac{\left[e_{M} \operatorname{sen} \mu\right]}{\left[e_{M} \cos \mu\right]}-1}{1,26-\frac{\left[e_{M} \operatorname{sen} \mu\right]}{\left[e_{M} \cos \mu\right]}}
$$

dove $\quad\left[e_{M} \operatorname{sen} \mu\right]=\sum e_{M} \operatorname{sen} \mu \quad \Leftrightarrow \quad\left[e_{M} \cos \mu\right]=\sum e_{M} \cos \mu$.

Con questo sistema l'errore su $\varphi$ viene notevolmente diminuito.

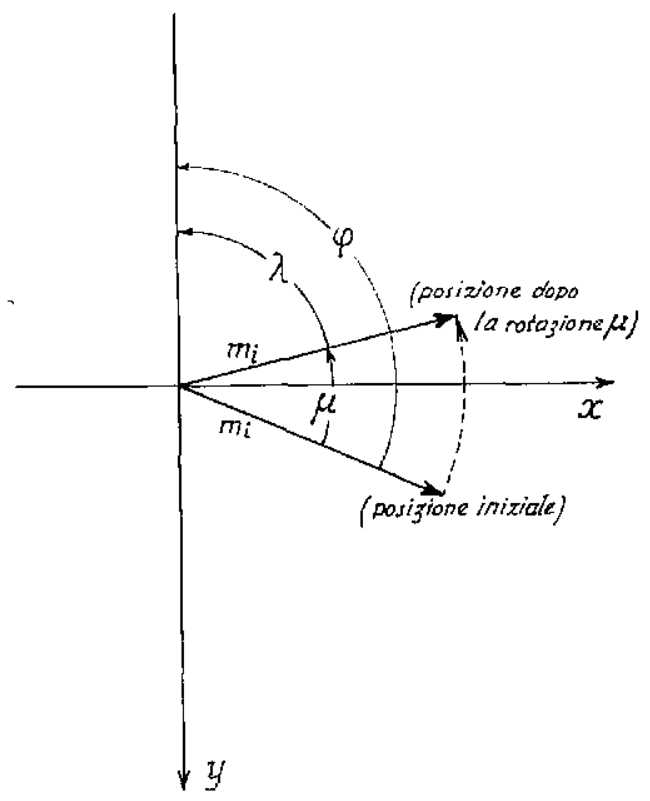

Fig. 8

Attualmente con questo apparato si sta procedendo, in collaborazione con 1'Istituto di Geologia dell'Università di Roma, al rilevamento della direzione dell'intensità di magnetizzazione delle colate lavicke dei vulcani laziali.

Istituto Nazionale di Geofisica. 


\section{BIBLIOGRAFIA}

(1) Nagata T., Rock Magnetism. Maruzen Tokio, 1953.

Konnigsberger J. (x., Tert. Magn., 43, 119, 229 (1938).

Fisher R. A., Proc. Roy. soc. Londom A-217, 295 (1953).

Runcons S. K.. Magnetization of Roeks, in "Handbuch der Physik". B. XLVII, 470, Springer-Verling, Berlin 1956.

Nagata T., Magnetic Properties of Rochs and Minerals, in "Handbuch der Physik ", B. XLIX/I, 248, springer-Verlag, Berlin 1966.

(2) Herzog R. F. K.-Tiscirter O., Rev. Sci. Instrum., 24, 1000 (1953). 